\title{
TRAVELING WAVES AND WEAK SOLUTIONS FOR AN EQUATION WITH DEGENERATE DISPERSION
}

\author{
DAVID M. AMBROSE AND J. DOUGLAS WRIGHT \\ (Communicated by James E. Colliander)
}

Abstract. We consider the following family of equations:

$$
u_{t}=2 u u_{x x x}-u_{x} u_{x x}+2 k u u_{x} .
$$

Here $k \neq 0$ is a constant and $x \in\left[-L_{0}, L_{0}\right]$. We demonstrate that for these equations there are compactly supported traveling wave solutions (which are in $H^{2}$ ), and the Cauchy problem (with $H^{2}$ initial data) possesses a weak solution which exists locally in time. These are the first degenerate dispersive evolution PDE where both of these features are known to hold simultaneously. Moreover, if $k<0$ or $L_{0}$ is not too large, the solution exists globally in time.

\section{INTRODUCTION}

Nonlinear dispersive partial differential equations, such as the nonlinear Schrödinger (NLS) equation or Korteweg-deVries (KdV) equations, have been the subject of intense mathematical scrutiny. The importance of linear dispersive effects in understanding the dynamics of these equations cannot be understated, particularly in establishing the existence of special solutions such as solitons and also in more general studies of the associated Cauchy problem. However, there are a number of dispersive equations in which the mechanism which generates the dispersive effects is itself nonlinear. For instance, consider the following class of quasilinear equations of $\mathrm{KdV}$ type studied in [6]:

$$
u_{t}=g_{3} u_{x x x}+g_{2} u_{x x}+g_{1} u_{x}+g_{0} u,
$$

where $g_{j}=g_{j}\left(\partial_{x}^{j} u, \ldots, u, x, t\right)$. Any dispersive character of these equations is due to the leading "Airy"-like term $g_{3} u_{x x x}$. In [6], the authors show that if the dispersive effects are, roughly speaking, uniform, then the equations are well-posed in Sobolev space and moreover exhibit the Kato-smoothing effects common to semilinear dispersive equations. Specifically they require $g_{3}$ to be uniformly bounded away from zero; that is, $\left|g_{3}\right| \geq c>0$. They also require $g_{2} \geq 0$ so that $g_{2} u_{x x}$ does not act like a backwards diffusion operator. Likewise, in the study of the well-posedness of quasilinear NLS equations in [10, there is an assumption that dispersive effects are uniform.

Nevertheless there are problems in which the dispersive effects may vanish. We call such equations degenerate dispersive equations, or DDE for short. The most famous example is the $K(m, n)$ family of equations developed in [18]:

$$
u_{t}=\left(u^{n}\right)_{x}+\left(u^{m}\right)_{x x x} .
$$

Received by the editors January 9, 2012.

2010 Mathematics Subject Classification. Primary 35Q53.

(C)2013 American Mathematical Society Reverts to public domain 28 years from publication 
Observe that these equations are in fact of the form (1) but do not satisfy (if $m \geq 2$ ) the hypotheses of [6]. Other commonly studied DDE are degenerate variations of NLS equations [23], Klein-Gordon equations [19], model equations for granular media [11, 15], the Camassa-Holm equation 1 [3], models for magma dynamics [22] and equations which arise in the numerical analysis of the KdV equation [9]. The most striking effect of degenerate dispersion is the existence of nonsmooth coherent structures with compact support. While there are numerous careful numerical studies ( $7,12,20,21)$ ) of compact traveling waves (i.e. compactons) and other types of compact structures, there are very few rigorous results concerning the existence and behavior of general solutions to the Cauchy problem $([2,7,8$,$) .$

In fact, recent results in [1] demonstrate that the degenerate Airy equation

$$
u_{t}=2 u u_{x x x}
$$

is in fact ill-posed in the sense that solutions do not depend continuously on the initial data. Equation (2), like $K(m, n)$, is of the form (11) but does not meet the "uniformly dispersive" hypothesis for well-posedness in [6]; it is perhaps the simplest equation which could be said to be degenerate and dispersive. The article [1] does not address the issue of existence and uniqueness of solutions to (2). It is natural, then, to ask if there are any DDE of the form of (1) for which some components of well-posedness are true for data in Sobolev spaces.

This paper answers this question in the affirmative. Specifically, we do so for the following family of equations:

$$
u_{t}=2 u u_{x x x}-u_{x} u_{x x}+2 k u u_{x} .
$$

Here $k \neq 0$ is a constant and $t>0$. We take $x \in X:=\left[-L_{0}, L_{0}\right]$ and assume $u$ satisfies periodic boundary conditions. We will be considering the Cauchy problem:

$$
u(x, 0)=\phi(x) \in H^{2} .
$$

By rescaling the time and space variables we can set $k= \pm 1$. Note this has the additional effect of changing the width of $X$, but since we view $L_{0}$ as a parameter in the problem, this change is not substantial. Note that (3) is a modification of the $K(2,2)$ equation: $v_{t}=\left(v^{2}\right)_{x x x}+\left(v^{2}\right)_{x}=2 v v_{x x x}+6 v_{x} v_{x x}+2 v v_{x}$.

We demonstrate (a) that (3) has compactly supported traveling wave solutions and (b) the Cauchy problem (with $H^{2}$ initial data) for (3) possesses a weak solution which exists locally in time. Equation (3) is the first degenerate dispersive evolution PDE where (a) and (b) are known to hold simultaneously. Since many studies of degenerate dispersive equations focus on interactions between compactly supported traveling waves, it is of fundamental importance that the Cauchy problem for initial data in the same class as the traveling waves be understood. In our case, our traveling wave solutions are in fact $H^{2}$, as is the case for the $K(m, n)$ equations.

In Section 2 we first prove some a priori estimates for smooth solutions of (3). Namely, we show that the $H^{2}$ norm is bounded uniformly in terms of the initial data, at least for finite times. In certain situations, the $H^{2}$ norm is bounded for all $t>0$. Subsequently we use those estimates to prove:

\footnotetext{
${ }^{1}$ The Camassa-Holm equation is both nonlocal and completely integrable. As such, it is not obvious how to generalize results which hold for it to other systems.
} 
Theorem 1. For $k= \pm 1$, for all $L_{0}>0$ and all $\phi \in H^{2}(X)$, there exists $T^{*}=$ $T^{*}\left(k, L_{0}, \phi\right) \in(0, \infty]$ so that for all $0<T<T^{*}$ there is a function

$$
u \in L^{2}\left(0, T ; H^{7 / 4}\right)
$$

which is a weak solution of (3). Specifically u satisfies (6) below. Additionally, if $k=-1$ or $L_{0}<\pi / \sqrt{2}$, then $T^{*}=+\infty$.

Remark 1 . The regularity we use above, $H^{7 / 4}$, is not sharp. Our result could be shown to hold in the Sobolev space $H^{2-\epsilon}$, where $\epsilon$ is any small and positive number. We choose $\epsilon=1 / 4$ primarily for concreteness.

Surprisingly, we find that for each wave speed $c>0$, there are multiple solitary wave solutions to (31) with a continuum of different amplitudes. As is the case for the $K(2,2)$ compactons, our traveling waves are not smooth, having a jump in the second derivative. There are several substantial differences between the $k=1$ and $k=-1$ cases. If $k=1$, there are solitary waves of arbitrarily large amplitude, though their width is bounded by a universal constant, independent of the domain size. On the other hand, if $k=-1$, the solitary waves have a maximal amplitude but can be as wide as the whole of $X$. In the case where $X=\mathbf{R}$, we find front solutions whose support is a half-line. In Section 3 we derive explicit formulae for the traveling wave solutions.

\section{EXISTENCE OF WEAK SOLUTIONS}

2.1. Energy estimates. In this section we prove several a priori estimates for (3). Let

$$
E[f]:=\int_{X}\left(\frac{1}{2} f^{2}(x)-\frac{5 k}{4} f_{x}^{2}(x)+\frac{1}{2} f_{x x}^{2}(x)\right) d x .
$$

Proposition 1. If $u$ is a sufficiently smooth solution of (3) with initial condition (4), we have for all $t$

$$
E[u(t)]=E[\phi] .
$$

Proof. Let

$$
\begin{gathered}
E_{0}(t):=\frac{1}{2} \int_{X} u^{2}(x, t) d x, \quad E_{1}(t):=-\frac{5 k}{4} \int_{X} u_{x}^{2}(x, t) d x, \\
E_{2}(t):=\frac{1}{2} \int_{X} u_{x x}^{2}(x, t) d x .
\end{gathered}
$$

Differentiating $E_{0}$ with respect to time, using the form of (3) given by (12) below, and then integrating by parts gives

$$
\begin{aligned}
\dot{E}_{0} & =\int_{X} u u_{t} d x \\
& =\int_{X} u\left(2 u u_{x x}-\frac{3}{2} u_{x}^{2}+k u^{2}\right)_{x} d x \\
& =-\int_{X} u_{x}\left(2 u u_{x x}-\frac{3}{2} u_{x}^{2}+k u^{2}\right) d x .
\end{aligned}
$$

Since $2 u u_{x} u_{x x}=u\left(u_{x}^{2}\right)_{x}$ and $u^{2} u_{x}=1 / 3\left(u^{3}\right)_{x}$, we can rewrite the last line above as

$$
-\int_{X}\left(u\left(u_{x}^{2}\right)_{x}-\frac{3}{2} u_{x}^{3}+\frac{k}{3}\left(u^{3}\right)_{x}\right) d x .
$$


Now, $\int_{X}\left(u^{3}\right)_{x}$ vanishes due to the periodic boundary conditions. A final integration by parts then gives

$$
\dot{E}_{0}=\frac{5}{2} \int_{X} u_{x}^{3} d x
$$

Similarly for $E_{1}$, we have after after integrating by parts in $x$ one time:

$$
\begin{aligned}
\dot{E}_{1} & =-\frac{5 k}{2} \int_{X} u_{x} u_{x t} d x \\
& =-\frac{5 k}{2} \int_{X} u_{x}\left(2 u u_{x x}-\frac{3}{2} u_{x}^{2}+k u^{2}\right)_{x x} d x \\
& =\frac{5 k}{2} \int_{X} u_{x x}\left(2 u u_{x x}-\frac{3}{2} u_{x}^{2}+k u^{2}\right)_{x} d x .
\end{aligned}
$$

Applying the derivative and multiplying out the integrand convert the last line above to

$$
\begin{aligned}
& \frac{5 k}{2} \int_{X} u_{x x}\left(-u_{x} u_{x x}+2 u u_{x x x}+2 k u u_{x}\right) d x \\
= & \frac{5 k}{2} \int_{X}\left(-u_{x} u_{x x}^{2}+2 u u_{x x} u_{x x x}+2 k u u_{x} u_{x x}\right) d x .
\end{aligned}
$$

We then use the fact that $2 u u_{x x} u_{x x x}=u\left(u_{x x}^{2}\right)_{x}$ to get

$$
\frac{5 k}{2} \int_{X}\left(-u_{x} u_{x x}^{2}+u\left(u_{x x}^{2}\right)_{x}+k u\left(u_{x}^{2}\right)_{x}\right) d x .
$$

A final integration by parts in the middle term results in

$$
\dot{E}_{1}=-5 k \int_{X} u_{x} u_{x x}^{2} d x-\frac{5 k^{2}}{2} \int_{X} u_{x}^{3} d x .
$$

Also, for $E_{2}$, we apply the time derivative, use (12) (see below) and integrate by parts one time:

$$
\begin{aligned}
\dot{E}_{2} & =\int_{X} u_{x x} u_{x x t} d x \\
& =\int_{X} u_{x x}\left(2 u u_{x x}-\frac{3}{2} u_{x}^{2}+k u^{2}\right)_{x x x} d x \\
& =-\int_{X} u_{x x x}\left(2 u u_{x x}-\frac{3}{2} u_{x}^{2}+k u^{2}\right)_{x x} d x .
\end{aligned}
$$

If we apply the two $x$-derivatives to the quantity in parentheses above we arrive at

$$
\begin{aligned}
& -\int_{X} u_{x x x}\left(-u_{x} u_{x x}+2 u u_{x x x}+2 k u u_{x}\right)_{x} d x \\
= & -\int_{X} u_{x x x}\left(-u_{x x}^{2}+u_{x} u_{x x x}+2 u u_{x x x x}+2 k u u_{x x}+2 k u_{x}^{2}\right) d x \\
= & -\int_{X}\left(-u_{x x x} u_{x x}^{2}+u_{x} u_{x x x}^{2}+2 u u_{x x x} u_{x x x x}+2 k u u_{x x} u_{x x x}+2 k u_{x}^{2} u_{x x x}\right) d x .
\end{aligned}
$$

Since $u_{x x x} u_{x x}^{2}=1 / 3\left(u_{x x}^{3}\right)_{x}$ and $u_{x} u_{x x x}^{2}+2 u u_{x x x} u_{x x x x}=\left(u u_{x x x}^{2}\right)_{x}$ we have

$$
-\int_{X}\left(-\frac{1}{3}\left(u_{x x}^{3}\right)_{x}+\left(u u_{x x x}^{2}\right)_{x}+2 k u u_{x x} u_{x x x}+2 k u_{x}^{2} u_{x x x}\right) d x .
$$


The first two terms are perfect derivatives and thus vanish upon integration due to the periodic boundary conditions. Observing that $2 u u_{x x} u_{x x x}=u\left(u_{x x}^{2}\right)_{x}$ and integrating by parts, the term $2 k u_{x}^{2} u_{x x x}$ gives

$$
\begin{aligned}
& -\int_{X}\left(2 k u u_{x x} u_{x x x}+2 k u_{x}^{2} u_{x x x}\right) d x \\
& =-\int_{X}\left(k u\left(u_{x x}^{2}\right)_{x}-4 k u_{x} u_{x x}^{2}\right) d x .
\end{aligned}
$$

A final integration by parts gives

$$
\dot{E}_{2}=5 k \int_{X} u_{x} u_{x x}^{2} d x
$$

Since $k= \pm 1$, we have $k^{2}=1$, and therefore

$$
\dot{E}=\dot{E}_{0}+\dot{E}_{1}+\dot{E}_{2}=0 \text {. }
$$

When $k=-1$, it is clear that $E^{1 / 2}$ is equivalent to the usual norm on $H^{2}$. On the other hand, when $k=1$, then $E_{1}$ is negative and $E^{1 / 2}$ is not necessarily equivalent to the $H^{2}$ norm. We have

Lemma 2. If $k=1$, then $E^{1 / 2}$ is equivalent to the usual norm on $H^{2}$ if and only if $0<L_{0}<\frac{\sqrt{2} \pi}{2}$.

Proof. Since $X=\left[-L_{0}, L_{0}\right]$, by Plancherel's theorem we have

$$
E(t)=2 L_{0} \sum_{n \in \mathbf{Z}}\left(\frac{1}{2}-\frac{5}{4}\left(\frac{n \pi}{L_{0}}\right)^{2}+\frac{1}{2}\left(\frac{n \pi}{L_{0}}\right)^{4}\right)\left|\hat{u}_{n}\right|^{2},
$$

where $\hat{u}_{n}=\left(2 L_{0}\right)^{-1} \int_{-L_{0}}^{L_{0}} u(x, t) e^{-i n \pi x / L_{0}} d x$ are the usual Fourier coefficients.

The polynomial

$$
\mu(n):=\frac{1}{2}-\frac{5}{4}\left(\frac{n \pi}{L_{0}}\right)^{2}+\frac{1}{2}\left(\frac{n \pi}{L_{0}}\right)^{4}
$$

has $\mu(n)>0$ if and only if $\left|n \pi / L_{0}\right|>\sqrt{2}$ or $\left|n \pi / L_{0}\right|<\sqrt{2} / 2$. Therefore, if there are no integers $n$ with $|n|$ in the set $\left[\sqrt{2} L_{0} / 2 \pi, \sqrt{2} L_{0} / \pi\right]$ we have $\mu(n)>0$ for all $n \in \mathbf{Z}$. This in turn implies that there exists $C>1$ such that for all $n \in \mathbf{Z}$,

$$
C^{-1}\left(1+\left(\frac{n \pi}{L_{0}}\right)^{4}\right)<\mu(n)<C\left(1+\left(\frac{n \pi}{L_{0}}\right)^{4}\right) .
$$

This then gives the equivalence of $E^{1 / 2}$ to the normal $H^{2}$ norm.

Thus we only need to determine when there are no integers $n$ with $|n|$ in the set $\left[\sqrt{2} L_{0} \pi / 2, \sqrt{2} L_{0} \pi\right]$. This happens precisely when $L_{0}<\sqrt{2} \pi / 2$. On the other hand, if $L_{0} \geq \sqrt{2} \pi / 2, \mu(n)$ is not strictly bounded above zero on the integers, and we cannot have the equivalence of the norms.

The following corollary gives a useful a priori estimate in situations where $E^{1 / 2}$ is not a norm on $H^{2}$. Let

$$
N[f]:=\frac{1}{2}\|f\|_{H^{2}}^{2}=\frac{1}{2}\left(\|f\|_{L^{2}}^{2}+\left\|f_{x x}\right\|_{L^{2}}^{2}\right) .
$$


Corollary 3. For $k= \pm 1$ and any $L_{0}>0$, we have for any sufficiently smooth solution of (3) :

$$
\frac{d}{d t} N[u(t)] \leq C N^{3 / 2}[u(t)]
$$

Proof. Since $N=N[u(t)]=E_{0}(t)+E_{2}(t)$, we have

$$
\begin{aligned}
\dot{N} & =\frac{5}{2} \int_{X} u_{x}^{3} d x+5 k \int_{X} u_{x} u_{x x}^{2} d x \\
& \leq C\left\|u_{x}\right\|_{L^{\infty}} \int_{X}\left(u_{x}^{2}+u_{x x}^{2}\right) d x \\
& \leq C\|u\|_{H^{2}}^{3} \\
& =C N^{3 / 2} .
\end{aligned}
$$

2.2. Construction of weak solutions. Let $\psi: X \times[0, \infty) \rightarrow \mathbb{R}$ be a compactly supported test function. Since $\psi$ is compactly supported, there exists $T>0$ such that $\psi(x, t)=0$ for all $t \geq T$. If we multiply (3) by $\psi$, integrate over $X \times[0, \infty)$ and subsequently integrate by parts in each of $x$ and $t$ as appropriate, we arrive at (6)

$\int_{0}^{T} \int_{X}\left[-\left(\partial_{t} \psi\right) u+\left(\partial_{x}^{3} \psi+k \partial_{x} \psi\right)\left(u^{2}\right)-\frac{7}{2}\left(\partial_{x} \psi\right)\left(\partial_{x} u\right)^{2}\right] d x d t=\int_{X} \psi(x, 0) \phi(x) d x$.

We say that $u \in L^{2}\left(0, T ; H^{7 / 4}\right)$ is a weak solution of (3) provided this relation holds for all test functions $\psi$. We now prove Theorem 1 .

Proof of Theorem 1 .

Step 1. Existence of approximate solutions. We begin by introducing a regularized version of equation (3). Let $\varepsilon>0$ be given, and let $\mathcal{J}_{\varepsilon}$ be a family of Friedrichs mollifiers (for instance, convolution with an appropriate smooth family of functions [14]). Then consider

$$
u_{t}=2 \mathcal{J}_{\varepsilon}\left(\left(\mathcal{J}_{\varepsilon} u\right)\left(\partial_{x}^{3} \mathcal{J}_{\varepsilon} u\right)\right)-\mathcal{J}_{\varepsilon}\left(\left(\partial_{x} \mathcal{J}_{\varepsilon} u\right)\left(\partial_{x}^{2} \mathcal{J}_{\varepsilon} u\right)\right)+2 k \mathcal{J}_{\varepsilon}\left(\left(\mathcal{J}_{\varepsilon} u\right)\left(\partial_{x} \mathcal{J}_{\varepsilon} u\right)\right)
$$

We use the same initial data as above, (4). It is sometimes useful to rewrite this as

$$
\partial_{t} u=\partial_{x}^{3} \mathcal{J}_{\varepsilon}\left(\mathcal{J}_{\varepsilon} u\right)^{2}-\partial_{x} \mathcal{J}_{\varepsilon}\left(\frac{7}{2}\left(\partial_{x} \mathcal{J}_{\varepsilon} u\right)^{2}\right)+k \partial_{x} \mathcal{J}_{\varepsilon}\left(\mathcal{J}_{\varepsilon} u\right)^{2}
$$

With the abundance of mollifiers above, it is clear that the right hand side of (7) is a bounded and continuous map from $H^{2}$ into itself. Thus the Picard theorem for ODEs on a Banach space applies: there is a solution of (7), denoted $u_{\varepsilon}(x, t)$, in $C^{1}\left(0, T_{\varepsilon} ; H^{2}\right)$ for some $T_{\varepsilon}>0$.

Step 2. Uniform time of existence. That the time of existence for $u_{\varepsilon}$ depends upon $\varepsilon$ is a problem, as we would like to take $\varepsilon \rightarrow 0$. Here we prove the following:

Lemma 4. For $k= \pm 1$ and $L_{0}>0$ there exists $T^{*}=T^{*}\left(k, L_{0}, \phi\right) \in(0, \infty]$ such that for all $T \in\left(0, T^{*}\right)$ and $\varepsilon>0$, the solutions $u_{\varepsilon}$ of (7) satisfy

$$
u_{\varepsilon} \in C^{1}\left(0, T ; H^{2}\right) \subset L^{2}\left(0, T ; H^{2}\right)
$$

and

$$
\partial_{t} u_{\varepsilon} \in C\left(0, T ; H^{-1}\right) \subset L^{2}\left(0, T ; H^{-1}\right) .
$$


These functions are bounded uniformly in $\varepsilon$ in these spaces. Finally, if $k=-1$ or $L_{0}<\pi / \sqrt{2}$, then $T^{*}=\infty$ for all $\phi$.

Proof. This lemma follows from the energy estimates derived in the previous section. The placement of mollifiers in (7) is done in such a way that these energy estimates carry over to the regularized equation. Specifically, we have for all $t \in\left[0, T_{\varepsilon}\right)$,

$$
E\left[u_{\varepsilon}(t)\right]=E[\phi]
$$

and

$$
\frac{d}{d t} N\left[u_{\varepsilon}(t)\right] \leq C N^{3 / 2}\left[u_{\varepsilon}(t)\right]
$$

Of course, the verification of (9) and (10) follows along lines similar to the proofs of Proposition 1 and Corollary [3] and we will suppress most of the details. The key point is to show that the mollifiers do not affect the structure of the energy argument.

If we set $F_{0}(t):=\frac{1}{2} \int_{X} u_{\varepsilon}^{2} d x$ and $v_{\varepsilon}=\mathcal{J}_{\varepsilon} u_{\epsilon}$, then

$$
\begin{aligned}
\dot{F}_{0} & =\int_{X} u_{\varepsilon} u_{\varepsilon, t} d x \\
& =\int_{X} u_{\varepsilon} \mathcal{J}_{\varepsilon}\left(2 v_{\varepsilon} v_{\varepsilon, x x}-\frac{3}{2} v_{\varepsilon, x}^{2}+k v_{\varepsilon}^{2}\right)_{x} d x \\
& =\int_{X}\left(\mathcal{J}_{\varepsilon} u_{\varepsilon}\right)\left(2 v_{\varepsilon} v_{\varepsilon, x x}-\frac{3}{2} v_{\varepsilon, x}^{2}+k v_{\varepsilon}^{2}\right)_{x} d x \\
& =\int_{X} v_{\varepsilon}\left(2 v_{\varepsilon} v_{\varepsilon, x x}-\frac{3}{2} v_{\varepsilon, x}^{2}+k v_{\varepsilon}^{2}\right)_{x} d x .
\end{aligned}
$$

In going from the second to the third line we have made use of the fact that $\mathcal{J}_{\varepsilon}$ is self-adjoint. Notice that the final line here is the same as that which appears in the expression for $\dot{E}_{0}$ in the proof of Proposition 1 except with $v_{\varepsilon}$ appearing instead of $u$. Thus the same steps used there lead us to

$$
\dot{F}_{0}=\frac{5}{2} \int_{X} v_{\varepsilon, x}^{3} d x
$$

In exactly the same fashion, if we set

$$
F_{1}(t):=-\frac{5 k}{4} \int_{X} u_{\varepsilon, x}^{2} d x \quad \text { and } \quad F_{2}(t):=\frac{1}{2} \int_{X} u_{\varepsilon, x x}^{2} d x
$$

then

$$
\dot{F}_{1}=-5 k \int_{X} v_{\varepsilon, x} v_{\varepsilon, x x}^{2} d x-\frac{5 k^{2}}{2} \int_{X} v_{\varepsilon, x}^{3} d x, \quad \dot{F}_{2}=5 k \int_{X} v_{\varepsilon, x} v_{\varepsilon, x x}^{2} d x .
$$

From this, (9) and (10) follow immediately.

If $k=-1$ or $L_{0}<\pi / \sqrt{2}$, Lemma 2 tells us that $E^{1 / 2}\left[u_{\varepsilon}(t)\right]$ is an equivalent norm for $H^{2}$. Thus (9) tells us that the $H^{2}$ norm of the solution cannot blow up at any time. Therefore, by the continuation theorem for autonomous ODEs on a Banach space, for any $0<T<\infty$ the solutions $u_{\varepsilon}$ of (7) exist on the interval $[0, T)$ and are in the space $C^{1}\left(0, T ; H^{2}(X)\right)$, bounded uniformly in $\varepsilon$.

On the other hand, if $k=1$ and $L_{0} \geq \pi / \sqrt{2}$, then $E^{1 / 2}$ is not a norm on $H^{2}$. Instead, we integrate the differential inequality (10) to find that there exists a time $T^{*}>0$, which is independent of $\varepsilon$, before which $N\left[u_{\varepsilon}(t)\right]=\frac{1}{2}\left\|u_{\varepsilon}\right\|_{H^{2}}^{2}$ must remain 
finite. That is, in this case, for any $0<T<T^{*}$ the solutions $u_{\varepsilon}$ of (7) exist on the interval $[0, T)$ and are in the space $C^{1}\left(0, T ; H^{2}(X)\right)$, bounded uniformly in $\varepsilon$.

That $\partial_{t} u_{\varepsilon}$ are bounded uniformly in $C\left(0, T ; H^{-1}\right)$ follows by examining the right hand side of (8). For instance, $u_{\varepsilon} \in H^{2}$ implies $u_{\varepsilon}^{2} \in H^{2}$, which in turn implies $\left(u_{\varepsilon}^{2}\right)_{x x x} \in H^{-1}$. Thus the first term on the right hand side is in $C\left(0, T ; H^{-1}\right)$. All the other terms are similar.

Step 3. Convergence of $u_{\varepsilon}$ to a weak solution. Fix $T<T^{*}$. The Aubin-Lions lemma states (see [4])

Lemma 5. Suppose that $B_{0}, B_{1}$, and $B_{-1}$ are three separable reflexive Banach spaces with $B_{1} \Subset B_{0}$ and $B_{0}$ continuously embedded in $B_{-1}$. Suppose that $f_{m}$ is a bounded sequence in $L^{p}\left(0, T ; B_{1}\right)$ and $\partial_{t} f_{m}$ is a bounded sequence in $L^{q}\left(0, T ; B_{-1}\right)$. Here $1<p, q<\infty$. Then there is a subsequence $f_{m^{\prime}}$ which converges in $L^{p}\left(0, T ; B_{0}\right)$.

$X$ is compact and thus $H^{2} \Subset H^{7 / 4} \Subset H^{-1}$. Therefore this lemma together with our Lemma 4 implies that there is a function $u$ such that

$$
u_{\varepsilon} \rightarrow u
$$

in $L^{2}\left(0, T ; H^{7 / 4}\right)$ as $\varepsilon \rightarrow 0$, along a subsequence. This function $u$ is the weak solution we are looking for, as we are about to see.

By multiplying (8) by $\psi$, a compactly supported test function, and then integrating in both space and time and integrating by parts, we find that the solutions $u_{\varepsilon}$ satisfy

$$
\begin{array}{r}
\int_{0}^{T} \int_{X}\left[-\left(\partial_{t} \psi\right) u_{\varepsilon}+\left(\partial_{x}^{3} \psi+k \partial_{x} \psi\right)\left(\mathcal{J}_{\varepsilon}\left(\mathcal{J}_{\varepsilon} u_{\varepsilon}\right)^{2}\right)-\left(\partial_{x} \psi\right)\left(\frac{7}{2} \mathcal{J}_{\varepsilon}\left(\partial_{x} \mathcal{J}_{\varepsilon} u_{\varepsilon}\right)^{2}\right)\right] d x d t \\
=\int_{X} \psi(x, 0) \phi(x) d x
\end{array}
$$

Since we have $u_{\varepsilon}$ converging strongly to $u \in L^{2}\left(0, T ; H^{7 / 4}\right)$, we have enough regularity to ensure that $\mathcal{J}_{\varepsilon}\left(\mathcal{J}_{\varepsilon} u_{\varepsilon}\right)^{2}$ converges strongly to $u^{2}$ and $\mathcal{J}_{\varepsilon}\left(\partial_{x} \mathcal{J}_{\varepsilon} u_{\varepsilon}\right)^{2}$ converges strongly to $\left(\partial_{x} u\right)^{2}$. We can therefore take the limit as $\varepsilon \rightarrow 0$ in (11), finding

$\int_{0}^{T} \int_{X}\left[-\left(\partial_{t} \psi\right) u+\left(\partial_{x}^{3} \psi+k \partial_{x} \psi\right)\left(u^{2}\right)-\frac{7}{2}\left(\partial_{x} \psi\right)\left(\partial_{x} u\right)^{2}\right] d x d t=\int_{X} \psi(x, 0) \phi(x) d x$.

Thus $u$ is a weak solution and we are done.

\section{EXISTENCE OF COMPACTLY SUPPORTED TRAVELING WAVES}

Note that (3) can be rewritten as

$$
u_{t}=\partial_{x}\left(2 u u_{x x}-\frac{3}{2} u_{x}^{2}+k u^{2}\right) .
$$

Making the traveling wave Ansatz $u(x, t)=Q(s)$ where $s=x-c t$, we find

$$
-c Q=2 Q \ddot{Q}-\frac{3}{2} \dot{Q}^{2}+k Q^{2} .
$$

We have integrated in $s$ one time. 
We rewrite (13) as a system

$$
\begin{aligned}
& \dot{Q}=P, \\
& \dot{P}=\frac{1}{2 Q}\left(-c Q-k Q^{2}+\frac{3}{2} P^{2}\right) .
\end{aligned}
$$

Our goal is to find a solution which is homoclinic to the origin and for which the duration of the trajectory is finite. We are able to find explicit formulae for such solutions. Notice that (14) has two equilibria: one at the origin (which is degenerate) and another at $Q=-c / k, P=0$.

Notice that when $P<0$ we have $\dot{Q}<0$. Therefore we can assert that a solution which lies in the lower half of the $Q P$-plane has a trajectory which is a graph over the $Q$ axis. That is,

$$
P(t)=f(Q(t))
$$

for an as yet unspecified function $f$. We differentiate this relationship and use (14):

$$
\dot{P}=f^{\prime}(Q) \dot{Q}=f^{\prime}(Q) P=f^{\prime}(Q) f(Q)=\frac{1}{2} \frac{d}{d Q}\left(f^{2}(Q)\right) .
$$

The second equation in (14) then becomes

$$
\frac{1}{2} \frac{d}{d Q}\left(f^{2}(Q)\right)=\frac{1}{2 Q}\left(-c Q-k Q^{2}+\frac{3}{2} f^{2}(Q)\right) .
$$

Letting $F=f^{2}$ and rearranging terms lead to the following ODE:

$$
F^{\prime}-\frac{3}{2 Q} F=-c-k Q \text {. }
$$

This can be solved explicitly for $F$ :

$$
F(Q)=2 c Q-2 k Q^{2}-\beta_{0} Q^{3 / 2},
$$

where $\beta_{0} \in \mathbf{R}$ is an arbitrary constant.

Thus the solution of (13) lies on the graph $P=-\sqrt{F(Q)}$, and therefore

$$
\dot{Q}=-\sqrt{2 c Q-2 k Q^{2}-\beta_{0} Q^{3 / 2}} .
$$

This equation can be solved explicitly, though the details vary depending on the sign of $k$.

3.1. Traveling pulses and fronts for $k=-1$. Separating variables and integrating (15) give

$$
\begin{aligned}
& \int_{A}^{Q(s)} \frac{d Q}{\sqrt{2 c Q+2 Q^{2}-\beta_{0} Q^{3 / 2}}}=-s \\
\Rightarrow & \sqrt{2} \ln \left(\frac{-\sqrt{2} \beta_{0} / 4+\sqrt{2 Q(s)}+\sqrt{2 c+2 Q(s)-\beta_{0} \sqrt{Q}(s)}}{-\sqrt{2} \beta_{0} / 4+\sqrt{2 A}+\sqrt{2 c+2 A-\beta_{0} \sqrt{A}}}\right)=-s .
\end{aligned}
$$

If we take $\beta_{0}$ so that $Q(0)=A>0$, we find

$$
Q(s)=\frac{1}{4 A}\left[c+A-(c-A) \cosh \left(\frac{\sqrt{2}}{2} s\right)\right]^{2} .
$$


This function diverges exponentially quickly as $s \rightarrow \infty$ and so does not immediately give the profile of a compactly supported traveling wave for (3). Notice that this function has two zeros when $0<A<c$ at

$$
s=L:= \pm \sqrt{2} \ln \left(\frac{c+A+2 \sqrt{c A}}{c-A}\right) .
$$

Since $Q(s)$ is nonnegative and in $C^{\infty}$, it follows that $\dot{Q}( \pm L)=0$ and $\ddot{Q}( \pm L)$ is finite and positive. Thus we can cut the function $Q$ off for $|s| \geq L$ and the resulting truncated function is still a solution of (13), since it is degenerate at $Q=0$.

That is to say, we have a pulse solution to (13) of the form

$$
Q(s)=Q_{c, A}^{-}(s):=\frac{\mathbf{1}_{L}(s)}{4 A}\left[A+c+(A-c) \cosh \left(\frac{\sqrt{2}}{2} s\right)\right]^{2} .
$$

Here $\mathbf{1}_{L}(s)$ is the characteristic function of $[-L, L]$. Clearly we require $L \leq L_{0}$.

We make the following observations about $Q_{c, A}^{-}$:

- The maximum of $Q_{c, A}^{-}$is $A$; thus for any speed $c>0$ there are compactly supported traveling waves with any amplitude $A \in(0, c)$.

- As $A \rightarrow c^{-}$, notice that $L \rightarrow+\infty$.

- $\lim _{s \rightarrow L^{-}} \frac{d^{2}}{d s^{2}} Q_{c, A}^{-}(s)=\lim _{s \rightarrow-L^{+}} \frac{d^{2}}{d s^{2}} Q_{c, A}^{-}(s)=c$.

There are also front solutions of (3) with speed $c$ if we allow $X=\mathbf{R}$. These correspond to the two heteroclinic solutions of (14) connecting the origin to the equilibrium at $(c, 0)$. These are given by

$$
Q_{c, \text { front }}^{-}(s)=\left\{\begin{array}{l}
c\left[1-e^{\frac{\sqrt{2}}{2}} s\right]^{2}, \text { if } s<0, \\
0, \text { otherwise }
\end{array}\right.
$$

and

$$
Q_{c, \text { back }}^{-}(s)=Q_{c, \text { front }}^{-}(-s)
$$

Observe that $\lim _{s \rightarrow 0^{-}} \frac{d^{2}}{d s^{2}} Q_{c, \text { front }}^{-}(s)=\lim _{s \rightarrow 0^{+}} \frac{d^{2}}{d s^{2}} Q_{c, b a c k}^{-}(s)=c$. We plot the various pulse and front solutions in Figures 1 and 2 .

3.2. Traveling pulses for $k=1$. We can likewise compute explicit formulae for pulses when $k=1$. The details are only slightly different than above, and so we only provide the end result:

$$
Q_{c, A}^{+}(s):=\frac{\mathbf{1}_{M}(s)}{4 A}\left[A-c+(A+c) \cos \left(\frac{\sqrt{2}}{2} s\right)\right]^{2} .
$$

Here $\mathbf{1}_{M}(s)$ is the characteristic function of $[-M, M]$ and

$$
M:=\sqrt{2} \arccos \left(\frac{c-A}{c+A}\right) .
$$

Clearly we require $M \leq L_{0}$.

We make the following observations about $Q_{c, A}^{+}$:

- The maximum of $Q_{c, A}^{+}$is $A$, and unlike when $k=-1$, there is no upper limit on $A$. For any speed $c>0$ there are compactly supported traveling waves with any amplitude $A>0$. 


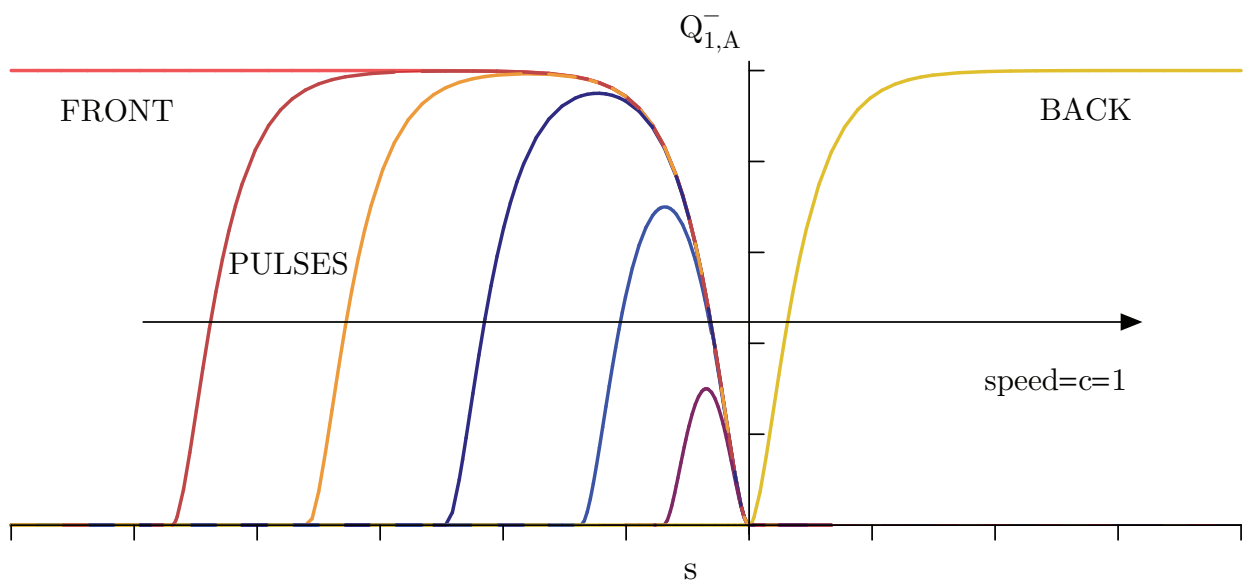

FiguRE 1. Profiles for traveling waves, including the front and back, of (3) when $k=-1$. Here, all waves move with the same speed $c=1$ but vary in amplitude. All traveling waves are asymptotic to the same parabola as they approach 0 . Also observe that pulses approach the front solution as their amplitude approaches 1.

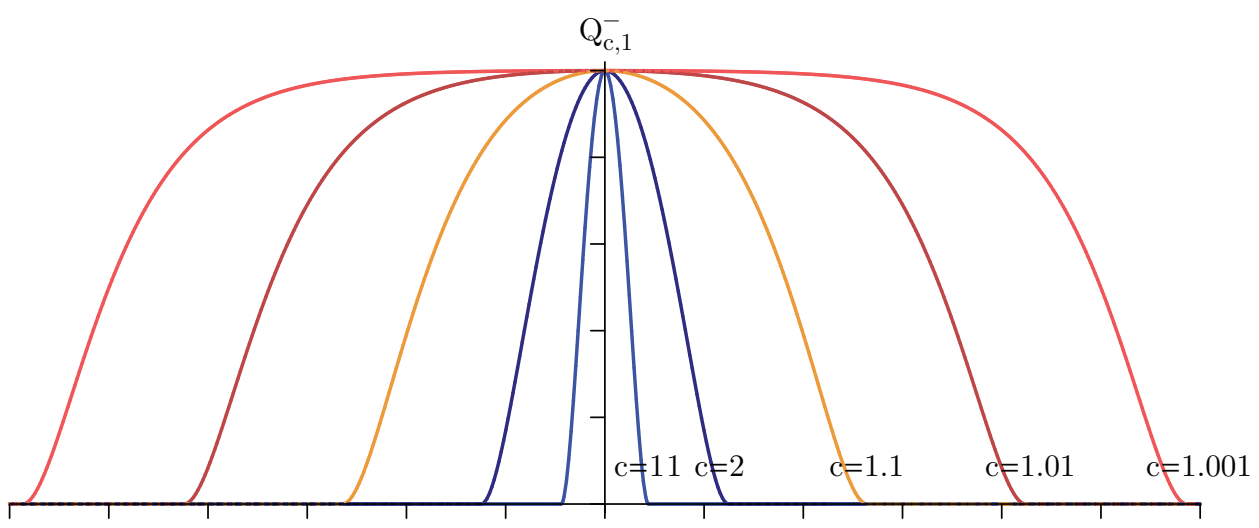

$\mathrm{S}$

Figure 2. Profiles for traveling waves of (3) when $k=-1$. Here, all waves have the same amplitude $A=1$ but vary in speed.

- As $A \rightarrow \infty$, notice that $M \rightarrow \sqrt{2} \arccos (-1)=\sqrt{2} \pi$ monotonically. Thus pulses have a maximum width in this setting.

- Similarly, for any amplitude $A>0$, there is a standing pulse $(c=0)$ which is of maximum width.

- As before $\lim _{s \rightarrow M^{-}} \frac{d^{2}}{d s^{2}} Q_{c, A}^{+}(s)=\lim _{s \rightarrow-M^{+}} \frac{d^{2}}{d s^{2}} Q_{c, A}^{+}(s)=c$.

We plot the various pulse solutions in Figures 3 and 4 


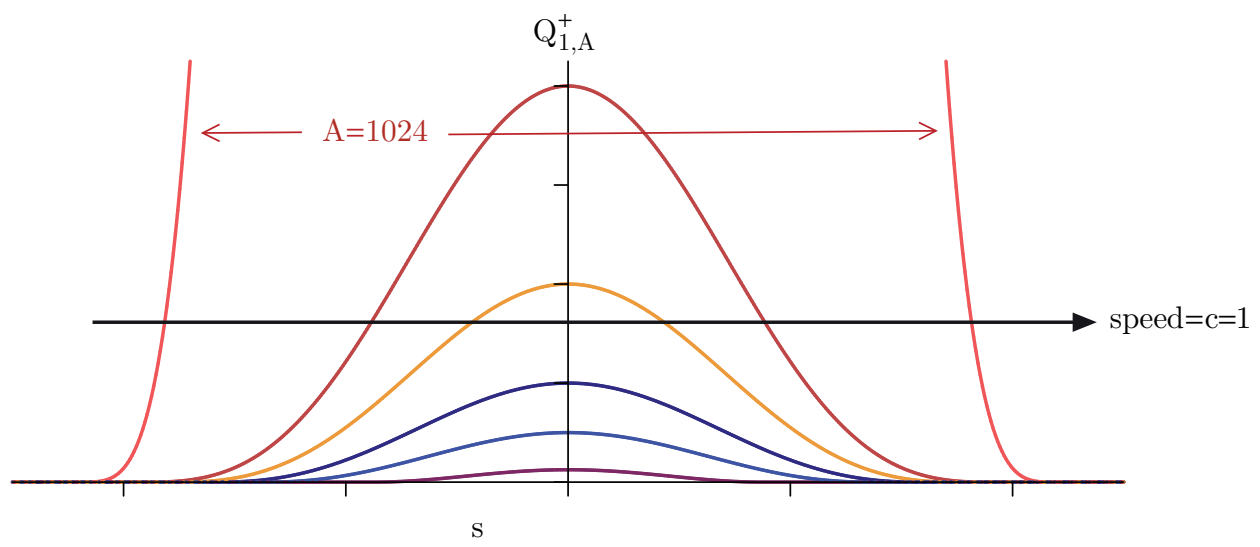

FiguRE 3. Profiles for traveling waves when $k=1$. Here, all waves move with the same speed $c=1$ but vary in amplitude. Observe that the large amplitude solution has width approaching $4.4 \sim$ $\sqrt{2} \pi$.

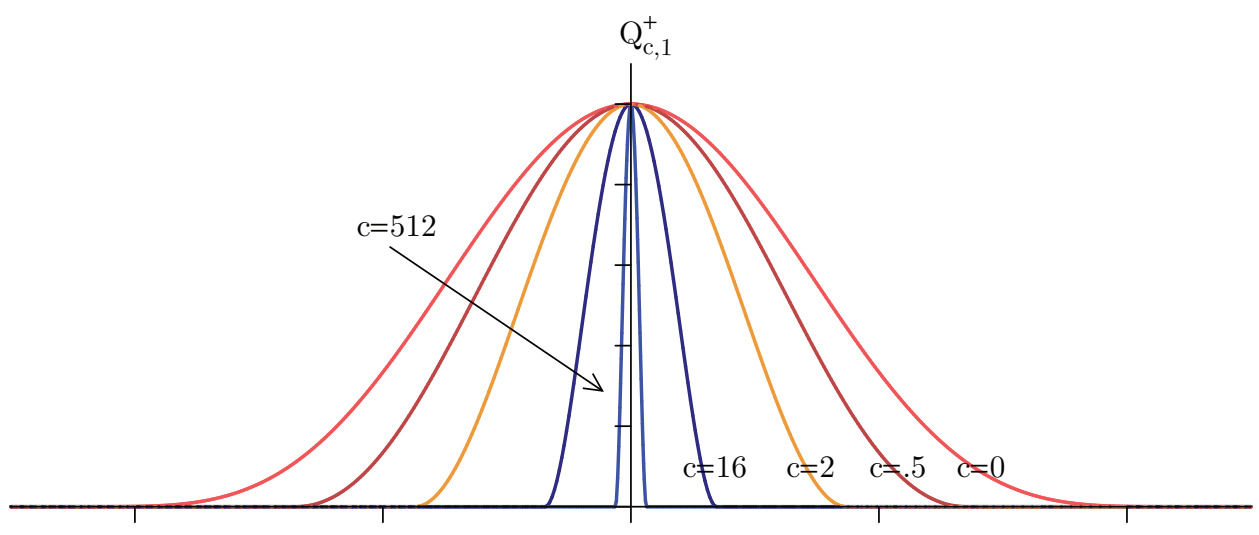

S

Figure 4. Profiles for a variety of traveling waves for the $k=1$ equation when $A=1$ and $c$ varies. Note that the $c=0$ wave has width exactly $4.4 \sim \sqrt{2} \pi$.

Remark 2. Note that for any $A \in \mathbf{R}$ and $c \in \mathbf{R}$,

$$
\tilde{Q}(s):=\frac{1}{4 A}\left[A-c+(A+c) \cos \left(\frac{\sqrt{2}}{2} s\right)\right]^{2}
$$

gives the profile of a periodic traveling wave for (3) when $k=1$ and the period of $\tilde{Q}$ is compatible with the width of the domain $X$. When $c>0$ and $A<0$, these periodic waves are negative valued and are strictly bounded away from zero; in particular, we have $\tilde{Q} \in\left[\frac{c^{2}}{4 A}, A\right]$. 


\section{ACKNOWLEDGEMENTS}

The first author was supported by National Science Foundation grants DMS1008387 and DMS-1016267. The second author was supported by National Science Foundation grants DMS-0807738, DMS-0908299 and DMS-1105635.

\section{REFERENCES}

[1] D. Ambrose, G. Simpson, J. Wright, and G. Yang. Ill-posedness of degenerate dispersive equations. Nonlinearity, 25 (2012), 2655-2680. MR.2967120

[2] David M. Ambrose and J. Douglas Wright, Preservation of support and positivity for solutions of degenerate evolution equations, Nonlinearity 23 (2010), no. 3, 607-620, DOI 10.1088/09517715/23/3/010. MR2586373 (2011i:35208)

[3] Roberto Camassa and Darryl D. Holm, An integrable shallow water equation with peaked solitons, Phys. Rev. Lett. 71 (1993), no. 11, 1661-1664, DOI 10.1103/PhysRevLett.71.1661. MR:1234453 (94f:35121)

[4] Peter Constantin and Ciprian Foias, Navier-Stokes equations, Chicago Lectures in Mathematics, University of Chicago Press, Chicago, IL, 1988. MR.972259 (90b:35190)

[5] Walter Craig and Jonathan Goodman, Linear dispersive equations of Airy type, J. Differential Equations 87 (1990), no. 1, 38-61, DOI 10.1016/0022-0396(90)90014-G. MR1070026 $(91 \mathrm{j}: 35238)$

[6] W. Craig, T. Kappeler, and W. Strauss, Gain of regularity for equations of KdV type, Ann. Inst. H. Poincaré Anal. Non Linéaire 9 (1992), no. 2, 147-186 (English, with French summary). MR 1160847 (93j:35153)

[7] J. de Frutos, M. Á. López Marcos, and J. M. Sanz-Serna, A finite difference scheme for the $K(2,2)$ compacton equation, J. Comput. Phys. 120 (1995), no. 2, 248-252, DOI 10.1006/jcph.1995.1161. MR.1349459

[8] V. A. Galaktionov, Nonlinear dispersion equations: smooth deformations, compactions, and extensions to higher orders, Zh. Vychisl. Mat. Mat. Fiz. 48 (2008), no. 10, 1859, DOI 10.1134/S0965542508100084 (Russian, with Russian summary); English transl., Comput. Math. Math. Phys. 48 (2008), no. 10, 1823-1856. MR2493771 (2009m:35050)

[9] Jonathan Goodman and Peter D. Lax, On dispersive difference schemes. I, Comm. Pure Appl. Math. 41 (1988), no. 5, 591-613, DOI 10.1002/cpa.3160410506. MR948073 (89f:65094)

[10] C. E. Kenig, G. Ponce, and L. Vega, The initial value problem for the general quasilinear Schrödinger equation, Recent developments in nonlinear partial differential equations, Contemp. Math., vol. 439, Amer. Math. Soc., Providence, RI, 2007, pp. 101-115, DOI 10.1090/conm/439/08466. MR2359023 (2009a:35235)

[11] David I. Ketcheson, High order strong stability preserving time integrators and numerical wave propagation methods for hyperbolic PDEs, ProQuest LLC, Ann Arbor, MI, 2009. Thesis (Ph.D.)-University of Washington. MR2713569

[12] Doron Levy, Chi-Wang Shu, and Jue Yan, Local discontinuous Galerkin methods for nonlinear dispersive equations, J. Comput. Phys. 196 (2004), no. 2, 751-772, DOI 10.1016/j.jcp.2003.11.013. MR.2054354 (2005d:65168)

[13] Yi A. Li, Peter J. Olver, and Philip Rosenau, Non-analytic solutions of nonlinear wave models, Nonlinear theory of generalized functions (Vienna, 1997), Chapman \& Hall/CRC Res. Notes Math., vol. 401, Chapman \& Hall/CRC, Boca Raton, FL, 1999, pp. 129-145. MR.1699882

[14] Andrew J. Majda and Andrea L. Bertozzi, Vorticity and incompressible flow, Cambridge Texts in Applied Mathematics, vol. 27, Cambridge University Press, Cambridge, 2002. MR.1867882(2003a:76002)

[15] M. Porter, C. Daraio, I. Szelengowicz, E. Herbold, and P. Kevrekidis. Highly nonlinear solitary waves in heterogeneous periodic granular media. Physica D 238 (2009).

[16] Philip Rosenau, Nonlinear dispersion and compact structures, Phys. Rev. Lett. 73 (1994), no. 13, 1737-1741, DOI 10.1103/PhysRevLett.73.1737. MR.1294558 (95f:35234)

[17] Philip Rosenau, Compact and noncompact dispersive patterns, Phys. Lett. A 275 (2000), no. 3, 193-203, DOI 10.1016/S0375-9601(00)00577-6. MR1807858 (2001k:35262)

[18] P. Rosenau and J. Hyman. Compactons: solitons with finite wavelength. Phys. Rev. Lett. 70 (1993), 564-567. 
[19] P. Rosenau and E. Kashdan. Emergence of compact structures in a Klein-Gordon model. Phys. Rev. Lett., (104), 2010.

[20] Francisco Rus and Francisco R. Villatoro, Padé numerical method for the RosenauHyman compacton equation, Math. Comput. Simulation 76 (2007), no. 1-3, 188-192, DOI 10.1016/j.matcom.2007.01.016. MR2392476(2009a:65222)

[21] Francisco Rus and Francisco R. Villatoro, Self-similar radiation from numerical Rosenau-Hyman compactons, J. Comput. Phys. 227 (2007), no. 1, 440-454, DOI 10.1016/j.jcp.2007.07.024. MR.2361530(2008k:65176)

[22] G. Simpson, M. Spiegelman, and M. I. Weinstein, Degenerate dispersive equations arising in the study of magma dynamics, Nonlinearity 20 (2007), no. 1, 21-49, DOI 10.1088/09517715/20/1/003. MR2285103 (2007i:86008)

[23] Zhenya Yan, Envelope compact and solitary pattern structures for the GNLS $(m, n, p, q)$ equations, Phys. Lett. A 357 (2006), no. 3, 196-203, DOI 10.1016/j.physleta.2006.04.032. MR2246313

Department of Mathematics, Drexel University, 3141 Chestnut Street, PhiladelPhia, Pennsylvania 19104

E-mail address: ambrose@math.drexel.edu

Department of Mathematics, Drexel University, 3141 Chestnut Street, PhiladelPhia, Pennsylvania 19104

E-mail address: jdoug@math.drexel.edu 